\title{
Chloroquine cardiomyopathy with conduction disorders
}

\author{
J-P Baguet, F Tremel, M Fabre
}

Department of

Cardiology and Internal Medicine, University Hospital, Grenoble, France J-P Baguet

F Tremel

Department of Internal Medicine, University Hospital M Fabre

Correspondence to: Dr J-P Baguet, Service de Médecine Interne et Cardiologie, Centre Hospitalier Universitaire de Grenoble, BP 217-38043 Grenoble Cedex 09, France.

Accepted for publication 10 July 1998

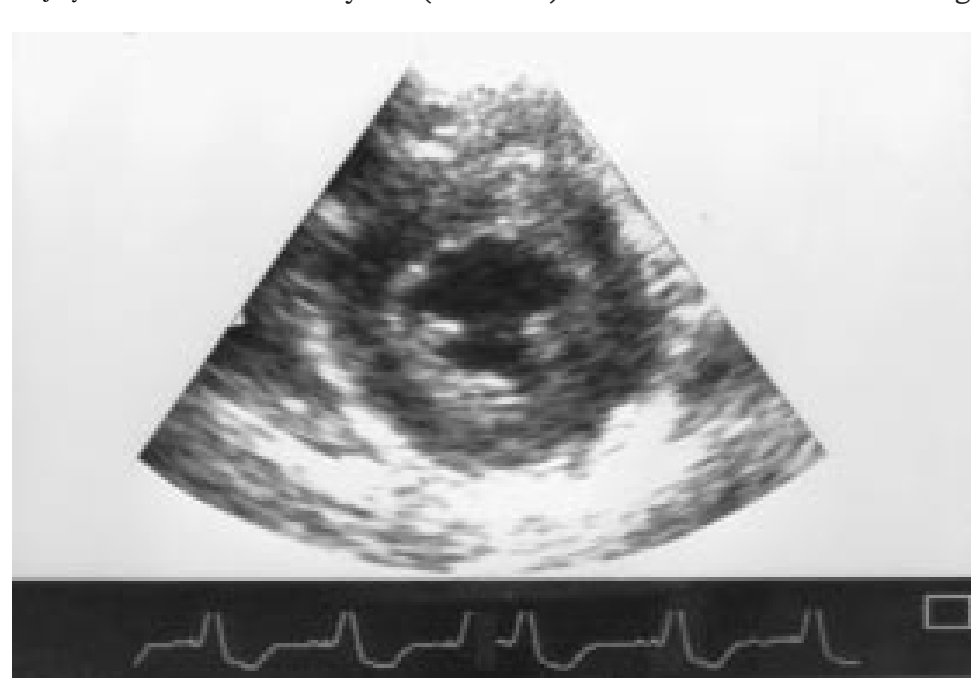

Figure 1 Cross sectional parasternal short axis view echocardiography suggesting biventricular hypertrophy.

A 58 year old female domestic worker was admitted to the department of general medicine because of diffuse abdominal pain and breathlessness on exertion. She also had fatigue and was found on examination to have right sided pleural and peritoneal effusions. These were confirmed by echography. History included pulmonary tuberculosis at the age of 18 and a diagnosis of disseminated lupus erythematosus (SLE) at the age of 20 with weakness, fever, lymphadenopathy, and a rash, which preceded nephropathy. She had been treated with corticosteroids for 15 years (1959-74); with azathioprine and cyclophosphamide for seven years (1959-66); with the antimalarial drugs hydroxychloquine for 25 years (1970-95) to a cumulative dose of $4380 \mathrm{~g}$

\begin{abstract}
A 58 year old woman on long term treatment with chloroquine for systemic lupus erythematosus presented with cardiac conduction disorders and heart failure with hypertrophic cardiomyopathy, which was confirmed by histology to be related to chloroquine toxicity. The heart failure improved on stopping chloroquine treatment.

(Heart 1999;81:221-223)
\end{abstract}

Keywords: chloroquine; cardiomyopathy; arrhythmias

. velocity of $70 \mathrm{~ms}$ without any other conduction anomaly. A DDD type pacemaker was implanted for security.

Transthoracic echocardiography (fig 1) suggested a diagnosis of biventricular cardiac hypertrophy with a left ventricular diameter of $37 \mathrm{~mm}$ and left wall thickness of $15 \mathrm{~mm}$, giving a relative wall thickness of 0.81 and a left ventricular mass index of $170 \mathrm{~g} / \mathrm{m}^{2}$. Left ventricular ejection fraction was $45 \%$ and the Appleton profile on the mitral valve Doppler was a restrictive type 3 . The right atrium was dilated and there was a small pericardial effusion but no valve lesions. The characteristic lesion was a white echogenic area in the walls especially in the septum. Because of this unusual hypertrophy on echography in the context of SLE, albeit seemingly inactive, an endocardial biopsy was done to eliminate secondary 


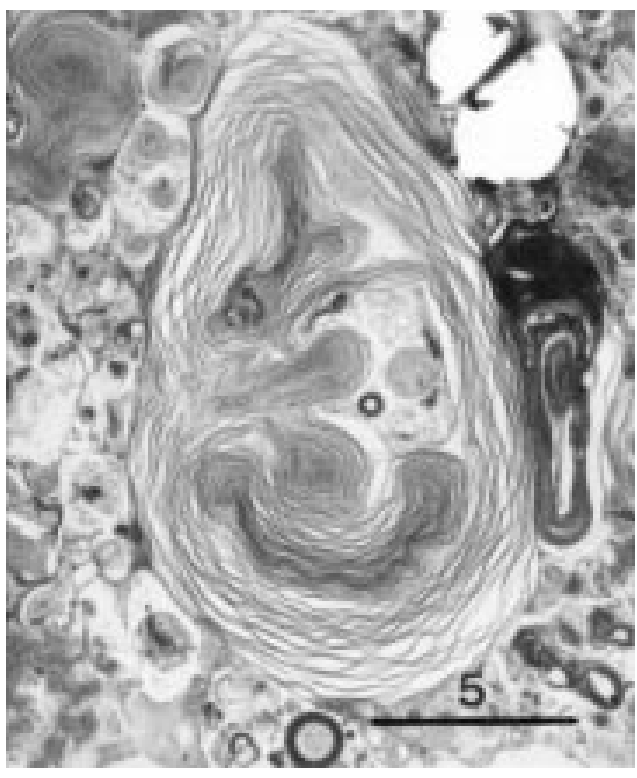

Figure 2 Lesions observed in cardiac and skeletal muscle fibres under ultrastructural examinations: pseudomyeloid structures.

amyloidosis, even though there was no biological evidence of progression of SLE.

Measurement of the right ventricular pressure (RVP) indicated a reduction in ventricular compliance (systolic RVP, $17 \mathrm{~mm} \mathrm{Hg}$; prediastolic, $2 \mathrm{~mm} \mathrm{Hg}$; end diastolic, $14 \mathrm{~mm} \mathrm{Hg}$ with a large a wave); mean right atrial pressure was $7 \mathrm{~mm} \mathrm{Hg}$. Microscopy of the myocardial biopsy specimen excluded amyloidosis, haemochromatosis, and glycogenesis but showed a vacuolisation in the myocardiocytes with disorganisation of the myofibrils and fibrosis in the connective tissues. Ultrastructural examination showed three types of lesions: a phagocytic necrosis giving dense heterogeneous bodies, pseudomyeloid formations, and curvilinear bodies (fig 2). In the absence of a storage disease such as ceroid-lipofuscinosis, these bodies are found with chloroquine toxicity; therefore, we diagnosed chloroquine cardiomyopathy. In the retina, examination of the fundus and the electroretinogram was normal. Electromyogram showed a mixed lesion of the nerve and muscle, and biopsy of the quadriceps showed a vacuolar necrosis with identical images to those of chloroquine myopathy on ultrastructural examination.

Chloroquine was stopped and treatment with angiotensin converting enzyme inhibitors and diuretics led to the disappearance of the signs of heart failure. Eight months after stopping the chloroquine treatment there have been no clinical or biological evidence of relapse in the inflammatory disorder. The patient has regained $8 \mathrm{~kg}$ in weight and has no evidence of heart failure. Muscle enzymes (creatine kinase and aldolase) are normal. Echocardiography showed left ventricular systolic function is normal with an ejection fraction of $70 \%$ and left ventricular mass index of $85 \mathrm{~g} / \mathrm{m}^{2}$. There remains a slight echodensity of the ventricle walls. The electromyogram shows only a few signs of myogenesis.

\section{Review of the literature}

Fewer than 20 cases of chloroquine cardiomyopathy have been reported. In our case, we observed an association between two cardiac complications of chloroquine toxicity: conduction disorder and hypertrophic cardiomyopathy. This pathology has been reported after prolonged treatment with chloroquine from seven months ${ }^{1}$ to 25 years, ${ }^{2}$ and may be more likely with renal failure. When there is a cardiac disorder, retinal involvement is inconsistent as is peripheral muscle involvement, although the histological appearance in muscle is identical.

ECHOCARDIOGRAPHIC ASPECTS

Clinical heart failure is a rare complication of long term treatment with chloroquine and the echocardiogram has rarely been recorded. As in our case, Cubero and colleagues ${ }^{2}$ reported a patient with biventricular myocardial hypertrophy with an echodense pattern in the walls and dilatation of both atria. McAllister and colleagues $^{3}$ also refer to biventricular hypertrophy in one case studied by echocardiography and another postmortem study, and similar findings are reported by Estes et al. ${ }^{1}$ In contrast, four reported cases with conduction disturbance had normal echocardiograms. ${ }^{4}$ The restrictive pattern found by us on Doppler examination was confirmed by haemodynamic data. This aspect of dip and plateau pressure tracing from the right ventricle has also been observed in a case with endomyocardial biopsies. $^{5}$

\section{CONDUCTION DISORDERS}

The association of severe conduction disorders and SLE treated with chloroquine is rare with 10 reported cases overall. The cause of the conduction problem in SLE is difficult to define as it may be related to the disease affecting the heart as well as the treatment. According to Godeau et $a l,{ }^{6}$ the incidence is $16 \%$ in a series of 112 cases with SLE, but the five patients with atrioventricular block were treated with chloroquine and two of these had cardiac involvement. The association of hypertrophic cardiomyopathy and severe atrioventricular block with histological evidence suggesting chloroquine toxicity confirms the iatrogenic origin of cardiac disease. Conduction disorders have been reported in acute overdose of chloroquine and in chloroquine treatment of malaria.

\section{HISTOLOGICAL ASPECTS}

In our patients, light microscopy showed cellular hypertrophy of myocardiocytes with heavily vacuolated cytoplasm and disorganisation of the myofibrillar architecture. There is neither inflammatory infiltrate evidence nor argument for vasculitis. Electron microscopy showed dense residual bodies with folded membranous aggregates and curvilinear bodies. This pattern is not seen in cardiac SLE without chloroquine treatment.

These lesions, except the curvilinear bodies, are seen in other storage diseases with lysosomal accumulation or with toxic reactions to amiodarone or perhexiline maleate. 
Experimental studies have shown that chloroquine impedes mitochondrial oxidative metabolism and is noxious to myocardial function in acute intoxication. Chronic treatment can lead to alteration of intracytoplasmic membranes that are stored in lysosomes and protected against enzyme attack. The intralysosomal phospholipases are in fact inactivated by the increase in $\mathrm{pH}$ induced by the chloroquine.

\section{Conclusion}

The occurrence of conduction disorders in a patient treated with chloroquine should raise the possibly of a toxic mechanism. Echocardiography should be used to look for cardiac hypertrophy and a biopsy specimen examined to confirm the toxicity of the treatment. Chloroquine treatment should be stopped on establishment of the diagnosis.

The few cases evaluated after an interval from stopping the treatment have had a favourable outcome with improvement in the left ventricular ejection fraction, ${ }^{1{ }^{4}}$ but most studies have shown persistence of clinical signs and histological lesions up to nine years after stopping treatment.

We thank P Mezin and F Labat-Moleur for histological analysis.

1 Estes ML, Ewing-Wilson D, Chou SM, et al. Chloroquine neuromyotoxicity. Clinical and pathological perspective. Am F Med 1987;82:447-55.

2 Cubero GI, Rodriguez Reguero JJ, Rojo Ortega JM. Restrictive cardiomyopathy caused by chloroquine. $\mathrm{Br}$ Heart f 1993;69:451-2.

3 McAllister HA Jr, Ferrans VJ, Hall RJ, et al. Chloroquineinduced cardiomopathy. Arch Pathol Lab Med 1987;111: induced

4 Verny C, De Gennes C, Sébastien P, et al. Heart conduction disorders in long-term treatment with chloroquine. Two new cases. Press Med 1992;21:800-4.

5 Ratliff NB, Estes ML, Myles JL, et al. Diagnosis of chloroquine cardiomyopathy by endomyocardial biopsy. $N$ Engl f Med 1987;316:191-3.

6 Godeau P, Guillevin L, Fechner J, et al. Disorders of conduction in lupus erythematosus: frequency and incidence in a group of 112 patients. Ann Med Interne 1981;132:234-40. 